\title{
Memory for Emotional Pictures in Patients with Alzheimer's Dementia: Comparing Picture-Location Binding and Subsequent Recognition
}

\author{
Marloes J. Huijbers, ${ }^{1}$ Heiko C. Bergmann, ${ }^{2}$ \\ Marcel G. M. Olde Rikkert, ${ }^{3}$ and Roy P. C. Kessels ${ }^{2,3,4}$ \\ ${ }^{1}$ Department of Psychiatry, Radboud University Nijmegen Medical Centre, Internal Post 966, P.O. Box 9101, \\ 6500 HB Nijmegen, The Netherlands \\ ${ }^{2}$ Donders Institute for Brain, Cognition and Behaviour, Radboud University Nijmegen, P.O. Box 9101, \\ 6500 HB Nijmegen, The Netherlands \\ ${ }^{3}$ Department of Geriatric Medicine and Alzheimer Centre Nijmegen, Radboud University Nijmegen Medical Centre, \\ P.O. Box 9101, 6500 HB Nijmegen, The Netherlands \\ ${ }^{4}$ Department of Medical Psychology, Radboud University Nijmegen Medical Centre, P.O. Box 9101, \\ 6500 HB Nijmegen, The Netherlands
}

Correspondence should be addressed to Marloes J. Huijbers, m.huijbers@psy.umcn.nl

Received 9 February 2011; Revised 8 June 2011; Accepted 9 June 2011

Academic Editor: Willem A. Van Gool

Copyright (๑) 2011 Marloes J. Huijbers et al. This is an open access article distributed under the Creative Commons Attribution License, which permits unrestricted use, distribution, and reproduction in any medium, provided the original work is properly cited.

Emotional content typically facilitates subsequent memory, known as the emotional enhancement effect. We investigated whether emotional content facilitates spatial and item memory in patients with Alzheimer's dementia (AD). Twenty-three AD patients, twenty-three healthy elderly, and twenty-three young adults performed a picture relocation task and a delayed recognition task with positive, negative, and neutral stimuli. AD patients showed a benefit in immediate spatial memory for positive pictures, while healthy young and older participants did not benefit from emotional content. No emotional enhancement effects on delayed item recognition were seen. We conclude that $\mathrm{AD}$ patients may have a memory bias for positive information in spatial memory. Discrepancies between our findings and earlier studies are discussed.

\section{Introduction}

There is abundant evidence that emotional events can be preserved in memory, even in patients with memory deficits. For example, amnesic patients could remember details of the terrorist attack of 9/11, 2001, while already suffering from profound anterograde amnesia at the time of the event [1]. This effect is generally referred to as the "emotional enhancement effect" [2]. Several experiments have shown that the emotional content of both pictures [3, 4] and words [57] facilitates subsequent memory for these items, although pictures evoke more arousal than words [8]. Furthermore, although enhancing effects of both valence (the subjective experience of a stimulus as positive or negative to a certain extent) [7] and arousal (physiological and psychological excitement evoked by the stimulus) have been demonstrated, most robust findings have been found when using highly arousing pictures (cf. [4]).

Fleming et al. [9] speculated that there may be an evolutionary advantage to not becoming overwhelmed by emotional stimuli at the time of the event, because it would interfere with survival instincts, but rather to recall and retain the information after a delay. Indeed, emotional arousal has been reported to impair task performance in a short-term memory task, but to enhance subsequent long-term memory for the emotional items compared with neutral ones [8]. Bower [10] suggested that emotional stimuli "consume" cognitive resources because of their attention-grabbing nature and 
therefore hamper short-term memory functioning. Evidence suggests that emotionally arousing stimuli attract attention already in the early perceptual stages. For example, both young and older adults notice threatening stimuli (e.g., pictures of dangerous animals) more quickly than neutral stimuli [11] and spend more time looking at them compared with neutral pictures [12]. Mather and colleagues [13] have demonstrated that short-term memory for picture locations decreased as the emotional arousal of the pictures increased, while long-term recall was better for the emotional items, compared with the neutral ones. Their findings support the idea of a trade-off between (impairment of) short-term memory and (enhancement of) long-term memory.

However, contrasting findings have been reported about the effects of emotional arousal on binding processes, that is, the integration of different aspects of information, for example, object and location. Mather and Nesmith [14] demonstrated that picture-location memory performance was consistently better for arousing than for neutral pictures. Other studies have also shown superior memory for the colour or location of emotional stimuli compared to that of neutral stimuli $[15,16]$. These findings are in contrast with the earlier report of Mather and colleagues [13] which showed impairment of picture-location memory for arousing pictures. To explain these contradictory findings, Mather proposed an object-based framework [17] with two components. The first component predicts that emotional arousal will enhance memory binding for within-object associations (e.g., object and location), but will impair or have no effect on between-object associations, in accordance with their recent findings [14]. The second component predicts that the attention-grabbing nature of emotional items will interfere with working memory when multiple bound representations have to be maintained simultaneously in working memory. In such situations, people could make conjunction errors (mixing up the features of different items) [18] because the limited working memory capacity is "overloaded" by the arousing information rather than elaborating on the association between picture and location. This latter suggestion could explain the results of the earlier study [13] in which four pictures of the same arousal level were shown rapidly (750 ms) and immediately after each other. In contrast, the studies showing enhanced location memory for arousing stimuli used a longer presentation duration with intervals between stimuli.

In patients with Alzheimer's dementia (AD), the effects of emotional content on memory are under debate. $\mathrm{AD}$ is characterized by profound memory impairment already in the early stages of the disease, caused by atrophy of the medial temporal lobe, including the hippocampus and the adjacent amygdala $[19,20]$. The degree of amygdala atrophy in early $\mathrm{AD}$ has been estimated at 36\% [21]. Abundant evidence suggests that the amygdala, particularly the basolateral part, plays a crucial role in arousal-based, emotional memory enhancement (for a review see [22]), since it interacts with memory formation subserved by the hippocampus (i.e., "emotional tagging"; [23]). In line with this, some studies have demonstrated that $\mathrm{AD}$ patients do not show memory enhancement for emotional pictures [24, 25]. In contrast, there are also studies that report relative sparing of memory for emotional verbal material in $\mathrm{AD}$ patients [9, 26-28]. These discrepancies may be due to differences in the methods and the materials that were used. For example, the studies that did not find emotional memory enhancement in $\mathrm{AD}$ $[24,25]$ used recognition tasks, whereas two of the studies reporting relative sparing of emotional memory $[9,27]$ used free recall tasks. Free recall may promote task engagement because it elicits a more active role for the participant, compared with answering only "yes" or "no" to presented stimuli. Possibly, task engagement is of particular importance in $\mathrm{AD}$ patients because they probably have more difficulty remembering the task instructions. Furthermore, Moayeri and colleagues [26] used a combination of visual and verbal material in a story format. This may promote a sense of personal involvement which may amplify the effect of emotion on memory. Indeed, personal involvement may be of particular importance in emotional memory enhancement in $\mathrm{AD}$ patients, since Mori and colleagues [28] have shown that $\mathrm{AD}$ patients who experienced the Kobe earthquake in 1995 were able to recall details about personal events during the earthquake quite well, whereas nonpersonal, factual knowledge was poorly recalled.

In sum, while there is evidence for an enhancing effect of emotional content on memory in general, different reports of emotional enhancement in picture-location binding exist. In addition, to our knowledge, there have been no studies to date that have assessed picture-location binding for emotional versus neutral stimuli in patients with $\mathrm{AD}$. The aim of this study is to investigate picture-location memory and subsequent (delayed) recognition memory for emotional stimuli (positive, negative, and neutral) in patients with mild to moderate $\mathrm{AD}$, compared with matched healthy older controls and younger controls. Because of the cognitive problems associated with $\mathrm{AD}$, we chose for an immediate cued recall paradigm, combined with a recognition (rather than free recall) paradigm, using visual stimuli. Participants were instructed to remember the locations of nine pictures during presentation and to relocate them immediately afterwards. Subsequently, an old/new delayed recognition paradigm was used to test long-term memory formation for the pictures presented in the first task. We predicted that emotional content would enhance picture-location binding in the healthy participants, consistent with the findings of Mather and Nesmith [14], Nashiro and Mather [29] and with the first component of the object-based framework [17]. In addition, we expected better recognition of emotional pictures by the control groups in the delayed recognition task, compared with neutral pictures. We hypothesized that none of these emotional enhancement effects would be found in AD patients, because of the suspected atrophy of the amygdala that diminishes its functioning and possibly undermines the effects of emotional arousal (cf. [25]). Accordingly, AD patients would both relocate and recognize the emotional pictures as accurately as the neutral pictures. Moreover, we expected the control groups to perform better than the $\mathrm{AD}$ patients, and the younger controls to perform better than the older controls. 


\section{Method}

2.1. Participants. Twenty-three patients with early AD (11 men) were recruited through the outpatient treatment clinic at the Geriatrics Department of Radboud University Nijmegen Medical Centre, the Netherlands. Patients could be included if they fulfilled the NINCDS-ADRDA criteria [30] for probable $\mathrm{AD}$ (mild to moderate stage; Clinical Dementia Ratings 1-2). All diagnoses were made in a multidisciplinary setting, supported by clinical history, physical examination, neuropsychological assessment, cerebrospinal fluid abnormalities, and/or neuroradiological findings (MRI $n=13$; CT $n=10$ ). Consequently, all patients also fulfilled the recently developed research criteria for $\mathrm{AD}$ [31]. Exclusion criteria were dementia of other types, other neurological complications (confirmed by MRI and CT scans), for example, cerebrovascular accident, traumatic brain injury, or Korsakoff's syndrome; psychiatric history, for example, major depressive disorder unrelated to $\mathrm{AD}$, anxiety disorder, or schizophrenia; and current signs of depression, that is, a score $>6$ on the Geriatric Depression Scale-Short Form (GDS-15; [32]).

Twenty-three healthy older participants matched for age and educational level $(11$ men $)$ were recruited from the investigators' social network and acquaintances. Most of them were living in a rural area or small village. All were community-dwelling older people who were living independently at home. People from different socioeconomical classes were recruited. Additionally, 23 younger control participants were recruited (10 men) from Radboud University Nijmegen (undergraduate college students). Exclusion criteria for the control groups were subjective cognitive complains (self-report), scores below 26 on the Mini-Mental State Examination (MMSE; [33]), a history of neurologic or psychiatric disease, and current depressive symptoms. All participants had normal or corrected to normal vision and hearing and were Dutch-speaking. Premorbid intelligence levels were estimated using the Dutch version of the National Adult Reading Task $[34,35]$. Table 1 shows the demographic variables, educational level, MMSE, GDS-15, and NART-IQ scores of the three groups. Educational level was based on the classification of Verhage [36] which ranges from 1 (less than primary school) to 7 (academic degree). The study was performed in accordance with the ethical standards laid down in the 1964 Declaration of Helsinki, and only competent AD patients were selected to participate [37]. The research protocol was approved by the human research ethics committee (CMO region Arnhem-Nijmegen no. 2008/113), and informed consents were obtained for all participants.

2.2. Procedure. For the picture relocation task, participants were seated in a comfortable chair, approximately 50 centimetres in front of a touch-sensitive screen (ELO AccuTouch 220, 21 inch) connected to a laptop (Compaq Presario V5000) containing Windows XP home edition. All pictures were presented using the Object Relocation software [38]. First, a $3 \times 3$ grid was presented $(18 \times 18 \mathrm{~cm})$ on the computer screen, in which nine pictures $(5.5 \times 5.5 \mathrm{~cm}$ each) were presented sequentially for 1500 milliseconds in a random-generated order, with a total duration of 13,500 milliseconds (see Figure 1 for a schematic representation of the picture relocation task). We used a presentation duration of $1500 \mathrm{~ms}$ instead of $1000 \mathrm{~ms}$, which was used by Mather and Nesmith [14], to take a possible age-related decline in information-processing speed in our older participant groups into account. One practice trial was presented with nine neutral pictures, and four experimental trials with three neutral, three positive, and three negative pictures. The pictures comprising each experimental trial had been randomized using computerized random generation, resulting in 12 versions of the task with different picture combinations (within the set of 36 pictures) across trials. Consequently, no more than four participants received the same version (two versions per group).

Participants were instructed to remember the locations of the pictures in order to relocate them afterwards. Immediately after presentation in the grid, five pictures appeared above and four below the empty grid and participants were asked to place the pictures at their original locations by first touching the picture and then touching the box in which they thought it had been presented, after which the picture appeared in that box. The boxes remained filled after the pictures were relocated. The relocation phase was self-paced with no time restriction, and participants were allowed to correct errors. Instructions (or parts of it) were repeated when necessary. Participants were not informed about the delayed recognition task.

The delayed recognition task was administered approximately ten minutes after the picture relocation task. During the period in between, only a nonemotional visuospatial task and a verbal intelligence task were administered, which did not interfere with the emotional picture-location task. Participants were again seated in front of the touch screen. The 36 pictures from the picture relocation task combined with 36 new distracter pictures ( 72 in total) were presented one by one in a randomized order. The pictures were shown twice as large as in the picture relocation task $(11 \times 11 \mathrm{~cm}$ each $)$. Participants were asked to indicate whether they had already seen the picture ("yes" response) or not ("no" response). The experimenter showed the next picture as soon as a clear answer was given. See Figure 2 for a schematic representation of the delayed recognition task. There was no time limit for answering, but the response procedure was forced-choice in case of hesitation. Participants were allowed to change their response to the previous item only. Instructions were repeated when necessary.

2.3. Stimuli. The pictures that were used in the experiment were selected from the International Affective Picture System (IAPS; [39]). This is a large set of standardized, emotionally evocative, internationally accessible, colour photographs that include contents across a wide range of semantic categories. It is developed and distributed by the NIMH Centre for Emotion and Attention (CSEA) at the University of Florida in order to provide standardized materials for researchers studying emotion and attention. Lang and colleagues [39] had students rate each picture on valence, arousal, and dominance, resulting in a mean value (between 1 and 9) for 
TABLE 1: Demographic variables, MMSE, GDS-15 and NART-IQ scores in the AD group, the older control group and the younger control group $(N=69)$.

\begin{tabular}{|c|c|c|c|c|}
\hline Variable & $\begin{array}{l}\text { AD patients } \\
\quad(n=23)\end{array}$ & $\begin{array}{l}\text { Older controls } \\
\qquad(n=23)\end{array}$ & Young controls $(n=23)$ & Significance \\
\hline Male/female & $11 / 12$ & $11 / 12$ & $10 / 13$ & Overall: $\chi^{2}=.117, P=.94$ \\
\hline NART-IQ $^{a}$ & $\begin{array}{c}88.13 \pm 22.01 \\
(\text { range 62-133) }\end{array}$ & $\begin{array}{l}91.30 \pm 16.87 \\
(\text { range } 72-135)\end{array}$ & $99.04 \pm 9.07($ range $80-115)$ & Overall: $F(2,66)=2.55, P=.09$ \\
\hline $\begin{array}{l}\text { Educational level } \\
(1-7)^{\mathrm{b}}\end{array}$ & $\begin{array}{l}4.0 \pm 1.6 \\
\text { (range } 1-7)\end{array}$ & $\begin{array}{l}4.3 \pm 1.0 \\
\text { (range } 2-7 \text { ) }\end{array}$ & $6.0 \pm 0.0$ & $\begin{array}{c}\text { AD versus older: } F(1,44)=.580 \\
P=.45\end{array}$ \\
\hline Age & $\begin{array}{c}77.2 \pm 4.5 \\
\text { (range } 68-88)\end{array}$ & $\begin{array}{c}78.9 \pm 5.4 \\
\text { (range 69-89) }\end{array}$ & $22.5 \pm 2.9($ range $19-30)$ & $\begin{array}{c}\text { AD versus older: } F(1,44)=1.34 \\
P=.25\end{array}$ \\
\hline MMSE score ${ }^{c}$ & $\begin{array}{c}22.0 \pm 3.9 \\
(\text { range } 15-28)\end{array}$ & $\begin{array}{c}28.7 \pm 1.2 \\
(\text { range } 27-30)\end{array}$ & N.a. & $\begin{array}{c}\text { AD versus older: } F(1,44)=45.81, \\
P<.001\end{array}$ \\
\hline GDS-15 score ${ }^{\mathrm{d}}$ & $\begin{array}{c}2.1 \pm 1.5 \\
\text { (range } 0-6)\end{array}$ & $\begin{array}{c}1.3 \pm 1.3 \\
\text { (range } 0-5 \text { ) }\end{array}$ & N.a. & $\begin{array}{c}\text { AD versus older: } F(1,44)=4.14 \\
P \leq .05\end{array}$ \\
\hline
\end{tabular}

Note. Numbers following \pm represent standard deviations from the mean (preceding number); n.a.: not applicable. ${ }^{\text {a}}$ NART: National Adult Reading Test; ${ }^{\mathrm{b}}$ Classification by Verhage [36]; ${ }^{\mathrm{c}}$ MMSE: Mini Mental State Examination; ${ }^{\mathrm{d}}$ GDS-15: Geriatric Depression Scale, 15 items.
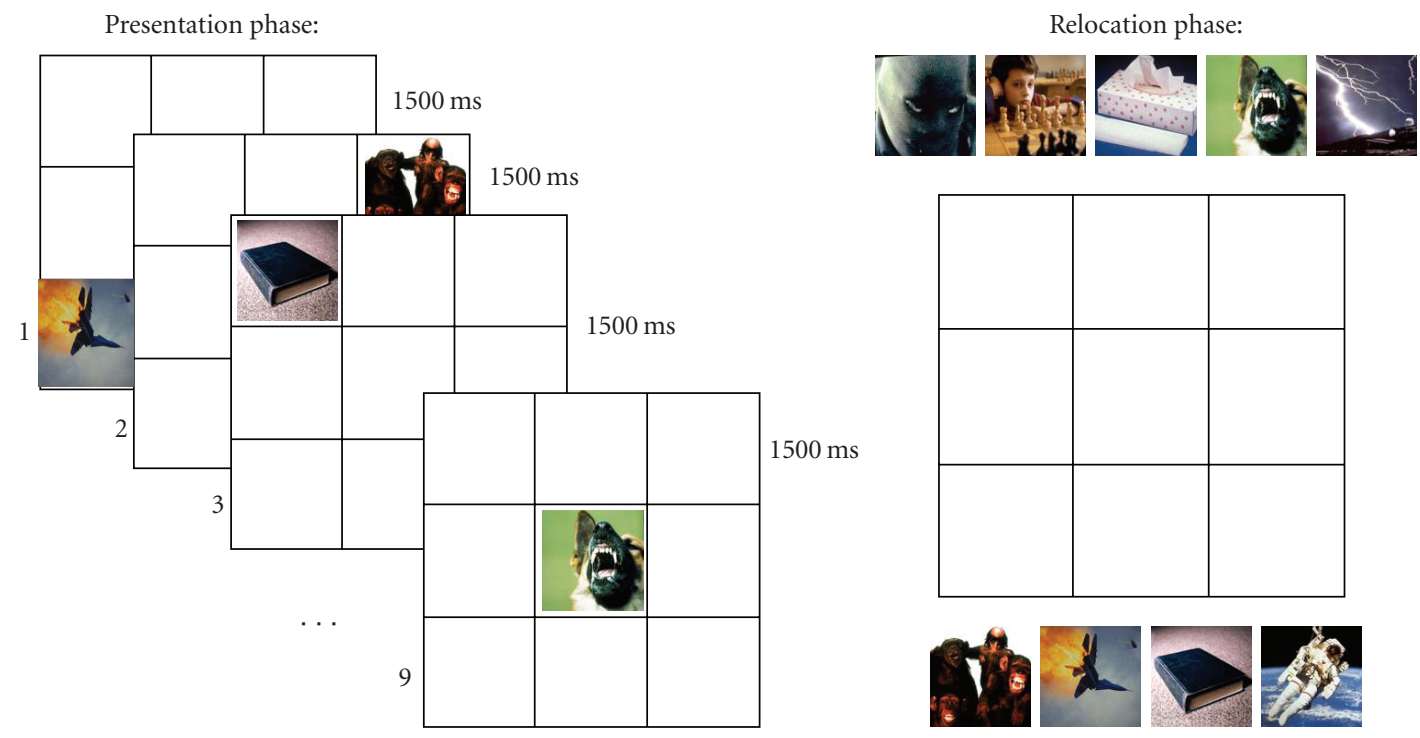

FIGURE 1: Schematic representation of the picture relocation task.

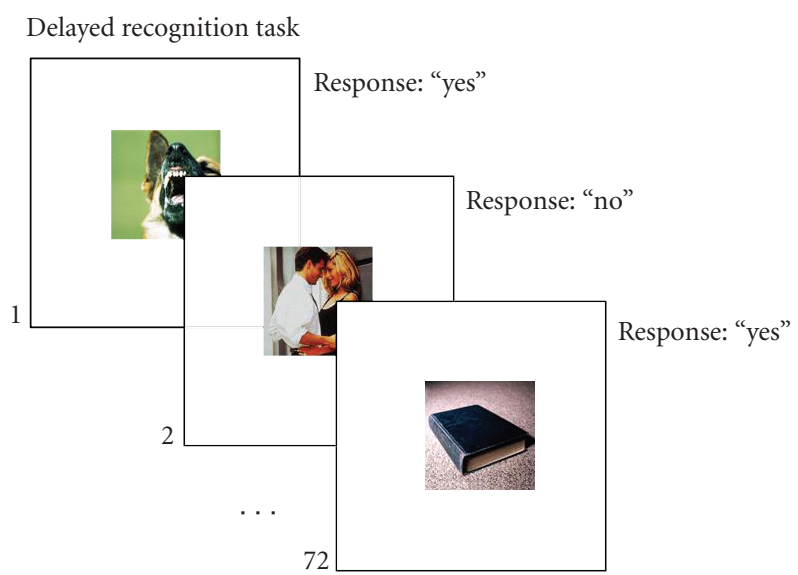

FIGURE 2: Schematic representation of the delayed recognition task. each dimension, using the Self-Assessment Manikin (SAM), an affective rating system devised by Lang et al. [39]. In this system, graphic figures depicting valence, arousal, and dominance dimensions on a continuously varying scale are used by participants to indicate their emotional reactions.

In this study, only the valence and arousal dimensions have been considered in the selection of a suitable picture set. For the picture relocation task, 36 pictures were chosen (12 neutral, 12 positive, and 12 negative). Nine additional neutral pictures were selected for the practice trial. The pictures were cut into squares (containing the essential visual information) in order to fit into the grid. For the delayed recognition task, 36 pseudorandomly selected distracter pictures of similar visual complexity that are visually distinct from the target pictures (12 neutral, 12 positive, and 12 negative) were added to the set. Statistical analysis was done to investigate 
the psychometric attributes of the total picture set, using the ratings from the study of Lang and colleagues [39]. Pairwise comparisons showed that there was a difference in arousal ratings between neutral $(m=2.56 \pm s d=0.34)$ and negative $(6.03 \pm 0.63)$ pictures $(P<.001)$ and between neutral $(2.56 \pm 0.34)$ and positive $(6.00 \pm 0.65)$ pictures $(P<$ $.001)$. Positive and negative pictures were equally arousing $(P=.82)$. Furthermore, there was a significant difference in valence across the positive, negative, and neutral picture groups (all $P$-values $<.001$ ), the positive pictures being rated as most pleasant $(7.24 \pm 0.66)$, with the negative pictures as least pleasant $(2.95 \pm 0.81)$, and the neutral pictures (5.18 \pm $0.46)$ in between. Analyses for the subset of the pictures used in the relocation task yielded highly similar results and represent the desired psychometric attributes. Analyses comparing the target versus distracter pictures showed no difference on arousal ( $m=6.2$ versus 5.9; $P=.33$ ) or valence $(2.9$ versus $3.0 ; P=.98)$ for the negative pictures, no difference on arousal $(m=5.9$ versus $6.1 ; P=.61)$ or valence ( $m=7.2$ versus $7.3 ; P=.77$ ) for the positive pictures and no difference on arousal ( $m=2.7$ versus $2.5 ; P=.16$ ) or valence ( $m=5.2$ versus $5.1 ; P=.77)$ for the neutral picture sets. Although the positive and negative pictures have higher ratings on the arousal dimension compared with neutral pictures, only the term "valence" is used in the current paper to distinguish the three emotion dimensions (i.e., positive, negative, and neutral).

\section{Results}

In all analyses, an alpha level of 0.05 was used, partial Eta squared $\left(\eta_{p}^{2}\right)$ was reported for analyses of variance, and Cohen's $d$ was reported for $t$-tests to indicate the effect sizes.

3.1. Picture Relocation. To evaluate performance on the picture relocation task, we first calculated the mean relocation distance (in $\mathrm{mm}$ ) between the original and chosen location of the three pictures of one emotional category within a trial (e.g., the mean relocation distance of the three positive pictures of trial 1). These scores were then averaged over the four experimental trials, resulting in three scores per participant (mean neutral, mean positive, and mean negative). Figure 3 shows the mean relocation distance scores for the three valence dimensions in the $\mathrm{AD}$ group and control groups.

A $3 \times 3$ repeated measures ANOVA was conducted with group (AD, old controls, young controls) as between-subject variable, valence (positive, negative, neutral) as within-subject variable, and relocation distance as dependent variable. There was a significant main effect of group $(F(2,66)=$ 104.51, $\left.P<.001, \eta_{\mathrm{p}}{ }^{2}=0.76\right)$. The mean relocation distance was lower (indicating better performance) for the young controls compared with the older controls $(F(1,44)=52.51$, $\left.P<.001, \eta_{\mathrm{p}}{ }^{2}=0.54\right)$ and compared with the AD patients $\left(F(1,44)=331.01, P<.001, \eta_{\mathrm{p}}{ }^{2}=0.88\right)$. The older controls also performed better than the $\mathrm{AD}$ patients $(F(1,44)=$ $\left.35.15, P<.001, \eta_{\mathrm{p}}{ }^{2}=0.44\right)$. No main effect of valence was found $\left(F(2,66)<1, P=.44, \eta_{\mathrm{p}}{ }^{2}=0.03\right)$. Averaged over the groups, positive, negative and neutral pictures were

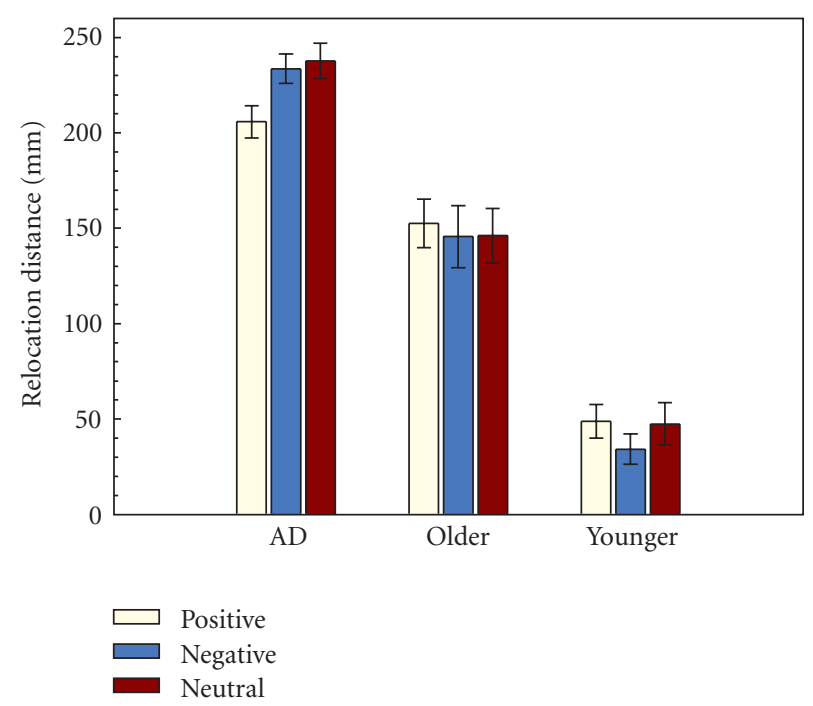

Figure 3: Mean relocation distance scores for positive, negative and neutral pictures in the $\mathrm{AD}$ group and control groups. Lower scores represent better performance. Error bars represent the standard error of the mean.

relocated equally well. However, there was a significant group $\times$ valence interaction $\left(F(4,130)=2.55, P<.05, \eta_{\mathrm{p}}{ }^{2}=0.07\right)$. Subsequent analyses showed that in the AD group, there was a difference in mean relocation time between the valence categories $\left(F(2,21)=4.47, P<.05, \eta_{\mathrm{p}}{ }^{2}=0.30\right)$. In the healthy older participants, no effect of valence on relocation was observed $\left(F(2,21)<1, P=.84, \eta_{\mathrm{p}}{ }^{2}=0.02\right)$. In the young controls, we found a trend towards an effect of valence $\left(F(2,21)=3.06, P=.07, \eta_{\mathrm{p}}{ }^{2}=0.27\right)$. Paired-sample $t$ tests showed that $\mathrm{AD}$ patients performed relatively better in relocating positive compared with neutral pictures $(t(22)=$ $2.82, P \leq .01, d=0.74)$ and negative pictures $(t(22)=2.48$, $P<.05, d=0.71)$. Relocation of neutral and negative pictures did not differ in the $\mathrm{AD}$ patients $(t(22)<1, P=.71$, $d=0.10)$. In the young control group, negative pictures were relocated better than neutral pictures $(t(22)=2.04$, $P \leq .05, d=0.29$ ) but not compared with positive pictures $(t(22)=1.96, P=.06, d=0.37)$. Relocation of positive and neutral pictures did not differ in the young control group $(t(22)<1, P=.87, d=0.03)$.

\subsection{Delayed Recognition}

3.2.1. Discriminability. For data analysis of the delayed recognition task, the proportion of hits (correctly responding "yes" to old pictures) and false alarms (incorrectly responding "yes" to new pictures) were calculated. Table 2 shows the mean number and proportion \pm standard error of hits and false alarms for positive, negative, and neutral stimuli in the AD and control groups. Participants' ability to correctly recognize old pictures and reject new ones (discriminability) was assessed in terms of the $d^{\prime}$ statistic [40], which reflects the probability ( $Z$-value) of hits minus the probability of false alarms. The $Z$-values for maximal and minimal recognition were set at 3.0 and -3.0 to 
TABLE 2: Mean number \pm standard deviation (proportion \pm standard deviation) of hits and false alarms for positive, negative, and neutral stimuli in the $\mathrm{AD}$ and control groups. Maximum per category $=12$.

\begin{tabular}{lcccc}
\hline Measure & Valence & AD group $(n=23)$ & Older controls $(n=23)$ & Young controls $(n=23)$ \\
\hline \multirow{3}{*}{ Hits } & Positive & $7.74 \pm 2.91(.64 \pm .24)$ & $10.00 \pm 1.76(.83 \pm .15)$ & $10.91 \pm 1.41(.91 \pm .12)$ \\
& Negative & $6.48 \pm 3.26(.54 \pm .27)$ & $9.61 \pm 2.61(.80 \pm .22)$ & $10.83 \pm 1.19(.90 \pm .10)$ \\
\hline \multirow{3}{*}{ False alarms } & Neutral & $8.57 \pm 2.86(.71 \pm .24)$ & $9.91 \pm 2.23(.83 \pm .19)$ & $10.74 \pm 1.05(.89 \pm .09)$ \\
& Positive & $4.00 \pm 3.26(.33 \pm .27)$ & $1.22 \pm 1.35(.10 \pm .11)$ & $0.70 \pm 1.11(.06 \pm .09)$ \\
& Negative & $3.70 \pm 2.84(.31 \pm .24)$ & $1.17 \pm 1.59(.10 \pm .13)$ & $0.48 \pm .59(.04 \pm .05)$ \\
& Neutral & $3.96 \pm 3.80(.33 \pm .32)$ & $0.74 \pm .86(.06 \pm .07)$ & $1.00 \pm 1.21(.08 \pm .10)$ \\
\hline
\end{tabular}

avoid artificially accentuated group differences that would be caused by a disproportionally high $Z$-value associated with a probability near 0.0 or 1.0 .

Figure 4 shows the recognition discriminability scores $\left(d^{\prime}\right)$ for the AD group and control groups. We performed a repeated-measures ANOVA with group as between-subject variable ( $\mathrm{AD}$, old controls, young controls), valence (positive, negative, neutral) as within-subject variable and discriminability $\left(d^{\prime}\right)$ as the dependent variable. There was a significant main effect of group $(F(2,66)=53.77, P<.001$, $\left.\eta_{\mathrm{p}}{ }^{2}=0.62\right)$. The young controls discriminated the old pictures from the new pictures more accurately than the older controls $\left(F(1,44)=4.49, P<.05, \eta_{\mathrm{p}}{ }^{2}=0.09\right)$ and the $\operatorname{AD}$ group $\left(F(1,44)=122.25, P<.001, \eta_{\mathrm{p}}{ }^{2}=0.74\right)$. The older controls also had higher discriminability scores than the $\mathrm{AD}$ patients $\left(F(1,44)=52.76, P<.001, \eta_{\mathrm{p}}{ }^{2}=\right.$ $0.55)$. There was no main effect of valence $(F(2,65)=1.33$, $\left.P=.27, \eta_{\mathrm{p}}{ }^{2}=0.04\right)$, but there was a significant group $\mathrm{x}$ valence interaction $\left(F(4,130)=2.56, P<.05, \eta_{\mathrm{p}}{ }^{2}=0.07\right)$. Subsequent analyses showed that in the $\mathrm{AD}$ group, there was a trend towards an effect of valence $(F(2,21)=3.31$, $\left.P=.06, \eta_{\mathrm{p}}{ }^{2}=0.24\right)$, while no effect of valence was found in the healthy older controls $(F(2,21)=1.10, P=.35$, $\left.\eta_{\mathrm{p}}{ }^{2}=0.10\right)$ or young controls $(F(2,21)=1.22, P=.32$, $\left.\eta_{\mathrm{p}}{ }^{2}=0.10\right)$. Post hoc comparisons showed that AD patients specifically recognized the neutral pictures better compared with negative pictures $(t(22)=2.64, P<.05, d=0.62)$, but not compared with positive pictures $(t(22)=1.69, P=.12$, $d=0.32$ ). There was no difference in the recognition of positive versus negative pictures in the AD group $(t(22)=$ 1.67, $P=.11, d=0.38$ ).

3.2.2. Response Criteria. In addition to the discriminability measure $d^{\prime}$, response criteria (c) were calculated [40]. Response criteria reflect the probability that a participant will respond "yes", irrespective of whether the stimulus is old or new. A lower value of $c$ indicates a more liberal response criterion (i.e., being more likely to say "yes", thereby increasing the number of hits and false alarms), while a higher value of $c$ indicates a more conservative response criterion.

Table 3 shows the response criterion values $(c)$ and standard errors for positive, negative, and neutral pictures in the $\mathrm{AD}$ and control groups. A repeated measures ANOVA with group ( $\mathrm{AD}$, old controls, young controls) as between-subject variable, valence (positive, negative, neutral) as within-subject variable, and response criterion $(c)$ as the dependent variable was conducted. There was no main effect of group

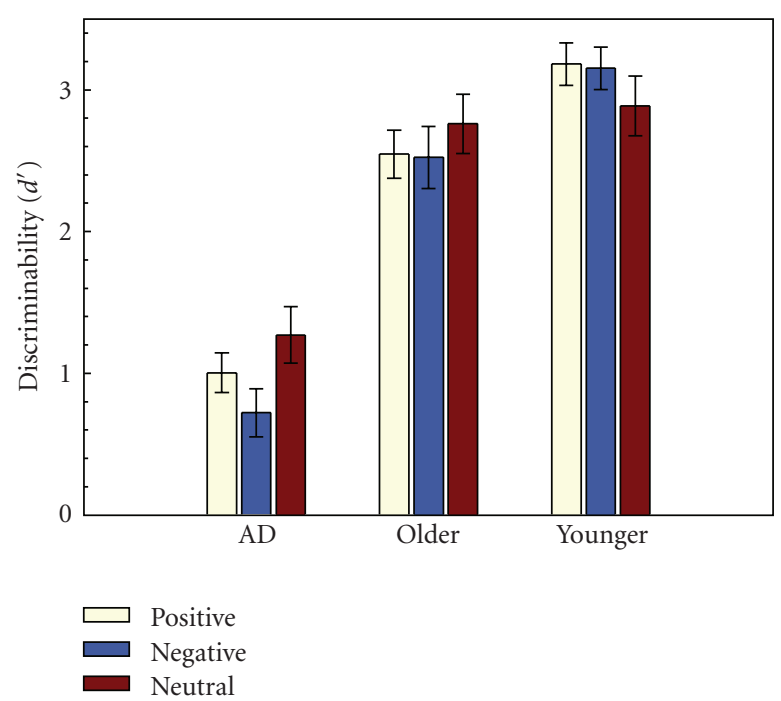

Figure 4: Recognition discriminability $\left(d^{\prime}\right)$ for positive, negative and neutral pictures in the AD and control groups. Error bars represent the standard error of the mean.

$\left(F(2,66)<1, P=.83, \eta_{\mathrm{p}}{ }^{2}=0.01\right)$, but there was a significant main effect of valence $\left(F(2,65)=3.60, P<.05, \eta_{\mathrm{p}}{ }^{2}=\right.$ $0.10)$. Paired-sample $t$-tests showed that response criteria were higher (more conservative) for negative compared with neutral pictures $(t(63)=2.63, P \leq .01, d=0.19)$, but not compared with positive pictures $(t(63)=1.55, P=.13, d=$ $0.16)$. Response criteria for positive versus neutral pictures also did not differ $(t(63)<1, P=.77, d=0.03)$. There was no group $\times$ valence interaction $(F(4,130)=1.22, P=.31$, $\left.\eta_{\mathrm{p}}{ }^{2}=0.04\right)$.

\section{Discussion}

In this study, the emotional enhancement effect was investigated using two different memory paradigms: a picture relocation task designed as a measure for visuospatial shortterm memory and an old/new delayed recognition task. We were interested in the performance of patients with $\mathrm{AD}$ on these tasks compared with matched, healthy older adults, and younger adults.

The mean relocation distance in the picture relocation task was larger (reflecting poorer performance) in the $\mathrm{AD}$ group compared with the healthy older group. The healthy older adults also performed worse than the younger controls. 
TABLE 3: Response criterion values (c) and standard errors for positive, negative and neutral pictures in the $\mathrm{AD}$ and control groups.

\begin{tabular}{lccc}
\hline Valence & $\begin{array}{c}\text { AD group } \\
(n=23)\end{array}$ & $\begin{array}{c}\text { Older controls } \\
(n=23)\end{array}$ & $\begin{array}{c}\text { Young controls } \\
(n=23)\end{array}$ \\
\hline$c$ positive & $0.04 \pm 0.18$ & $0.11 \pm 0.10$ & $0.01 \pm 0.09$ \\
$c$ negative & $0.19 \pm 0.17$ & $0.15 \pm 0.10$ & $0.09 \pm 0.06$ \\
$c$ neutral & $-0.04 \pm 0.17$ & $0.14 \pm 0.08$ & $0.02 \pm 0.05$ \\
\hline
\end{tabular}

These results indicate both an impairment associated with $\mathrm{AD}$ and an age-related impairment in picture-location memory. Short-term memory binding deficits have already been reported in $\mathrm{AD}$ patients compared with healthy older persons [41]. In general, older persons seem to have more difficulty binding features together $[29,42,43]$ and/or maintaining bound presentations in short-term memory $[29,44]$ than younger persons. Nashiro and Mather [29] showed that both young and older adults recalled more arousing than neutral pictures (item memory), whereas only young adults had better location memory for arousing compared with neutral pictures. Possibly, binding deficits in older people overrule the emotional enhancement effects that were seen at the level of item memory. In our sample, there was no main effect of valence on picture relocation. Healthy participants relocated positive, negative, and neutral pictures equally well, except for a trend in the young control group indicating that they might have benefited from negative content during the relocation task. In contrast with our expectations, however, $\mathrm{AD}$ patients showed an emotional enhancement effect for positive pictures, compared with neutral and negative pictures. Indeed, there is evidence for a shift towards positive information in attention and memory, as people age [4547]. In our study, such a positivity bias was only seen in the $\mathrm{AD}$ group. A similar memory bias for positive pictures in $\mathrm{AD}$ patients has been reported by Hamann et al. [25] using an immediate free recall task. A possible speculation could be that amygdala atrophy particularly affects the effect of negative arousal on memory, relatively sparing positive emotional memory. One of the limitations of this study is that we could not collect volumetric data on amygdala atrophy. In future studies, it would be interesting to investigate whether amygdala atrophy correlates with a memory bias for positive information. Given the scarce amount of literature on this specific issue, more research is needed to replicate current and previous findings.

In the delayed recognition task, $\mathrm{AD}$ patients showed impairment in discriminating previously seen (old) pictures from new (distracter) pictures compared with healthy control participants. Similarly, older controls had lower discriminability scores than younger controls. Again, these results indicate an impairment associated with $\mathrm{AD}$ as well as an age-related impairment in discriminability. Averaged over the groups, pictures with positive, negative, and neutral content were discriminated equally well. Our hypothesis that emotional arousal would facilitate long-term recognition memory in the healthy control groups (e.g., $[3,4]$ ) was not supported. However, a significant interaction effect was found. A trend indicated that only $\mathrm{AD}$ patients' recognition memory was affected by valence. More specifically, they showed superior recognition of neutral compared with negative pictures. This finding does not support our hypothesis that AD patients' recognition would not be affected by emotional content. Instead, it suggests the opposite of emotional enhancement (an "emotional impairment effect") in AD patients, when considering neutral versus negative pictures. This is in accordance with the findings of Hamann et al. [25] who also reported better (although not significant) recognition of neutral compared with negative and positive pictures in $\mathrm{AD}$ patients.

In all our groups, more conservative response criteria were observed for negative compared with neutral pictures. In other words, all groups were less inclined to respond "yes" to negative pictures than to neutral pictures in the recognition task. In the AD group, this conservative response criterion for negative stimuli may account for our finding that negative pictures were recognized least accurately. Possibly, the responses to the pictures during the recognition task were based on the patients' valence estimates rather than their memory for these items, for example, because the $\mathrm{AD}$ patients had difficulty remembering what kind of response was expected from them (i.e., recognition of items) due to diminished task engagement.

The absence of a memory enhancement effect in the long-term memory task needs further investigation. A possible explanation is that the process of memory consolidation requires a longer period than ten minutes, which we used in this study. Different timescales for the consolidation process have been proposed, ranging from thirty minutes to several months [2]. Although enhancing effects of emotional content may only be found after a minimum of thirty minutes, we chose for ten minutes because we expected that otherwise, the cognitive limitations in our study population would lead to floor effects. Furthermore, the recognition task was based on incidental learning rather than intentional learning. Although retention performance can be lessened by incidental learning, we did not find indications for floor effects. Only in the $\mathrm{AD}$ group, discriminability for negative pictures did not differ from chance level, but the other valence categories were discriminated above chance level.

There are other methodological issues to consider as well. First, our pictures may not have been sufficiently arousing. One could question whether the use of student ratings for the selection of the picture set is suitable to the older participant groups. There are studies reporting that age does not affect subjective arousal and valence ratings to IAPS pictures (e.g., $[25,45])$ or that older participants have even higher arousal ratings and more extreme valence ratings [48]. However, physiological reactions such as heart rate deceleration and corrugator electromyogram (related to a facial muscle used during frowning) in response to highly arousing IAPS pictures (including erotica and threatening faces) have been found to be weaker in older compared with younger participants [48]. In our sample of older participants, physiological reactions to the emotional pictures may have been even weaker, since we chose not to use highly arousing pictures because of our vulnerable participant group. This age-related decrease in physiological reactions to emotional stimuli 
could provide an explanation for the absence of an emotional enhancement effect in the recognition task. However, it does not explain the absence of emotional enhancement in our young participants.

Second, we presented the pictures for $1500 \mathrm{~ms}$ without interstimulus intervals during the relocation task. It is possible that the relatively fast presentation rate required participants to keep multiple bound representations simultaneously in mind. According to the second component of the objectbased framework [17], this may have caused interference with working memory and may have led to conjunction errors. The absence of emotional enhancement may also have been caused by information overload in our study. The impairing effect that was found in the study of Mather and colleagues using multiple arousing stimuli [13] may not have been observed in our study since our trials consisted of a combination of neutral, negative, and positive pictures.

A third limitation is that we did not ask people to describe what they saw. They may not always have understood the content of the picture, which may have decreased the emotional impact of the picture. The pictures may also have been too small to elicit strong effects on emotional arousal. This could account for the absence of an emotional enhancement effect in both the relocation task and the recognition task.

Finally, it is difficult to know whether the performance of the $\mathrm{AD}$ patients in the relocation task was at floor level, since we do not know which responses reflect the worst picturelocation memory. For example, would relocating a picture in the opposite corner of the $3 \times 3$ grid be a more accurate response than the middle box? The first response reflects at least the association between the picture and a corner, while the box in the middle would share less similarities with the correct location. The latter would result in a lower mean distance score (reflecting a better performance). This is even more complicated by the fact that participants had to take into account all nine pictures in a composite configuration, in which one error always implied another. Therefore, future studies aimed at replication should directly compare tasks based on multiple choice answers (e.g., as was done by Mather and Nesmith [14]) with tasks similar to the one used in the current study, in which participants have to recall the original location.

In conclusion, this study examined picture-location memory for emotional stimuli in $\mathrm{AD}$ patients. Our results showed that $\mathrm{AD}$ patients benefited from positive content in short-term picture-location memory, while both young and older healthy adults did not show enhancement for emotionally arousing information. Additionally, no effects of emotional picture content on delayed recognition were observed in this sample. Our results are not in agreement with other findings on emotional memory in healthy adults, suggesting that the emotional enhancement effect is sensitive to differences in task requirements and levels of cognitive functioning. In future studies, it would be interesting to investigate the attentional processes involved in emotional memory, for example, using eye-tracking methods. Furthermore, inclusion of volumetric amygdala measures in $\mathrm{AD}$ patients would provide further insight into the neurocog- nitive processes involved in object location memory for emotional information.

\section{Acknowledgments}

The authors wish to thank William van Aalst and Anja Bos for their great assistance in recruiting patients for this research project. Furthermore, they thank all participants for their time and effort. This study was supported by a VIDI Innovational Grant from the Netherlands Organization for Scientific Research (NWO, no. 452-08-005), awarded to R. P. C. Kessels. This study was carried out at the Radboud University Nijmegen Medical Centre, Department of Geriatric Medicine.

\section{References}

[1] I. Candel, M. Jelicic, H. Merckelbach, and A. Wester, "Korsakoff patients' memories of September 11, 2001," Journal of Nervous and Mental Disease, vol. 191, no. 4, pp. 262-265, 2003.

[2] S. Hamann, "Cognitive and neural mechanisms of emotional memory," Trends in Cognitive Sciences, vol. 5, no. 9, pp. 394400, 2001.

[3] S. B. Hamann, T. D. Ely, S. T. Grafton, and C. D. Kilts, "Amygdala activity related to enhanced memory for pleasant and aversive stimuli," Nature Neuroscience, vol. 2, no. 3, pp. 289293, 1999.

[4] M. M. Bradley, M. K. Greenwald, M. C. Petry, and P. J. Lang, "Remembering pictures: pleasure and arousal in memory," Journal of Experimental Psychology: Learning, Memory, and Cognition, vol. 18, no. 2, pp. 379-390, 1992.

[5] K. A. Nielson, D. Yee, and K. I. Erickson, "Memory enhancement by a semantically unrelated emotional arousal source induced after learning," Neurobiology of Learning and Memory, vol. 84, no. 1, pp. 49-56, 2005.

[6] T. M. Libkuman, C. L. Stabler, and H. Otani, "Arousal, valence, and memory for detail," Memory, vol. 12, no. 2, pp. 237-247, 2004.

[7] E. A. Kensinger and S. Corkin, "Memory enhancement for emotional words: are emotional words more vividly remembered than neutral words?" Memory and Cognition, vol. 31, no. 8, pp. 1169-1180, 2003.

[8] E. A. Kensinger and S. Corkin, "Effect of negative emotional content on working memory and long-term memory," Emotion, vol. 3, no. 4, pp. 378-393, 2003.

[9] K. Fleming, S. H. Kim, M. Doo, G. Maguire, and S. G. Potkin, "Memory for emotional stimuli in patients with Alzheimer's disease," American Journal of Alzheimer's Disease and other Dementias, vol. 18, no. 6, pp. 340-342, 2003.

[10] G. H. Bower, "How might emotions affect learning?" in The Handbook of Emotion and Memory: Research and Theory, S. Christianson, Ed., pp. 245-268, Lawrence Erlbaum Associates, New Jersey, NJ, USA, 1992.

[11] M. Mather and M. R. Knight, "Angry faces get noticed quickly: threat detection is not impaired among older adults," Journals of Gerontology-Series B Psychological Sciences and Social Sciences, vol. 61, no. 1, pp. P54-P57, 2006.

[12] K. S. LaBar, M. M. Mesulam, D. R. Gitelman, and S. Weintraub, "Emotional curiosity: modulation of visuospatial attention by arousal is preserved in aging and early-stage Alzheimer's disease," Neuropsychologia, vol. 38, no. 13, pp. 1734-1740, 2000. 
[13] M. Mather, K. J. Mitchell, C. L. Raye, D. L. Novak, E. J. Greene, and M. K. Johnson, "Emotional arousal can impair feature binding in working memory," Journal of Cognitive Neuroscience, vol. 18, no. 4, pp. 614-625, 2006.

[14] M. Mather and K. Nesmith, "Arousal-enhanced location memory for pictures," Journal of Memory and Language, vol. 58, no. 2, pp. 449-464, 2008.

[15] C. B. Hadley and D. G. MacKay, "Does emotion help or hinder immediate memory? Arousal versus priority-binding mechanisms," Journal of Experimental Psychology: Learning, Memory, and Cognition, vol. 32, no. 1, pp. 79-88, 2006.

[16] S. Doerksen and A. P. Shimamura, "Source memory enhancement for emotional words," Emotion, vol. 1, no. 1, pp. 5-11, 2001.

[17] M. Mather, "Emotional arousal and memory binding. An object-based framework," Perspectives on Psychological Science, vol. 2, pp. 33-52, 2007.

[18] M. T. Reinitz and S. L. Hannigan, "False memories for compound words: role of working memory," Memory and Cognition, vol. 32, no. 3, pp. 463-473, 2004.

[19] D. Chan, N. C. Fox, R. I. Scahill et al., "Patterns of temporal lobe atrophy in semantic dementia and Alzheimer's disease," Annals of Neurology, vol. 49, no. 4, pp. 433-442, 2001.

[20] K. K. Zakzanis, S. J. Graham, and Z. Campbell, "A metaanalysis of structural and functional brain imaging in dementia of the Alzheimer's type: a neuroimaging profile," Neuropsychology Review, vol. 13, no. 1, pp. 1-18, 2003.

[21] S. Lehericy, M. Baulac, J. Chiras et al., "Amygdalohippocampal MR volume measurements in the early stages of Alzheimer disease," American Journal of Neuroradiology, vol. 15, no. 5, pp. 929-937, 1994.

[22] J. L. McGaugh, "The amygdala modulates the consolidation of memories of emotionally arousing experiences," Annual Review of Neuroscience, vol. 27, pp. 1-28, 2004.

[23] G. Richter-Levin and I. Akirav, "Emotional tagging of memory formation-in the search for neural mechanisms," Brain Research Reviews, vol. 43, no. 3, pp. 247-256, 2003.

[24] J. Abrisqueta-Gomez, O. F. A. Bueno, M. G. M. Oliveira, and P. H. F. Bertolucci, "Recognition memory for emotional pictures in Alzheimer's patients," Acta Neurologica Scandinavica, vol. 105, no. 1, pp. 51-54, 2002.

[25] S. B. Hamann, E. S. Monarch, and F. C. Goldstein, "Memory enhancement for emotional stimuli is impaired in early Alzheimer's disease," Neuropsychology, vol. 14, no. 1, pp. 8292, 2000.

[26] S. E. Moayeri, L. Cahill, Y. Jin, and S. G. Potkin, "Relative sparing of emotionally influenced memory in Alzheimer's disease," NeuroReport, vol. 11, no. 4, pp. 653-655, 2000.

[27] R. R. Schultz, C. C. de Castro, and P. H. F. Bertolucci, "Memory with emotional content, brain amygdala and Alzheimer's disease," Acta Neurologica Scandinavica, vol. 120, no. 2, pp. 101110, 2009.

[28] E. Mori, M. Ikeda, N. Hirono, H. Kitagaki, T. Imamura, and T. Shimomura, "Amygdalar volume and emotional memory in Alzheimer's disease," American Journal of Psychiatry, vol. 156, no. 2, pp. 216-222, 1999.

[29] K. Nashiro and M. Mather, "How arousal affects younger and older adults' memory binding," Experimental Aging Research, vol. 37, no. 1, pp. 108-128, 2011.

[30] G. McKhann, D. Drachman, and M. Folstein, "Clinical diagnosis of Alzheimer's disease: report of the NINCDS-ADRDA work group under the auspices of Department of Health and Human Services Task Force on Alzheimer's disease," Neurology, vol. 34, no. 7, pp. 939-944, 1984.
[31] B. Dubois, H. H. Feldman, C. Jacova et al., "Research criteria for the diagnosis of Alzheimer's disease: revising the NINCDSADRDA criteria," The Lancet Neurology, vol. 6, no. 8, pp. 734746, 2007.

[32] J. I. Sheikh and J. A. Yesavage, "Geriatric Depression Scale (GDS): recent evidence and development of a shorter version," Clinical Gerontologist, vol. 5, no. 1-2, pp. 165-173, 1986.

[33] M. F. Folstein, S. E. Folstein, and P. R. McHugh, “'Mini mental state. A practical method for grading the cognitive state of patients for the clinician," Journal of Psychiatric Research, vol. 12, no. 3, pp. 189-198, 1975.

[34] H. E. Nelson, National Adult Reading Test (NART): Test Manual, NFER-Nelson, Windsor, UK, 1982.

[35] B. Schmand, J. Lindeboom, and F. Van Harskamp, NLV: Nederlandse Leestest Voor Volwassenen: Handleiding, Swets \& Zeitlinger, Lisse, The Netherlands, 1992.

[36] F. Verhage, Intelligentie en Leeftijd: Onderzoek bij Nederlanders van Twaalf tot Zevenenzeventig Jaar, Van Gorcum, Assen, The Netherlands, 1964.

[37] O. Meulenbroek, M. Vernooij-Dassen, R. P. C. Kessels et al., "Informed consent in dementia research. Legislation, theoretical concepts and how to assess capacity to consent," European Geriatric Medicine, vol. 1, no. 1, pp. 58-63, 2010.

[38] R. P. C. Kessels, A. Postma, and E. H. F. De Haan, "Object Relocation: a program for setting up, running, and analyzing experiments on memory for object locations," Behavior Research Methods, Instruments, and Computers, vol. 31, no. 3, pp. 423-428, 1999.

[39] P. J. Lang, M. M. Bradley, and B. N. Cuthbert, "International affective picture system (IAPS): instruction manual and affective ratings," Tech. Rep. A-6, The Center for Research in Psychophysiology, University of Florida, Gainesville, Fla, USA, 2005.

[40] D. M. Green and J. A. Swets, Signal Detection Theory and Psychophysics, John Wiley \& Sons, New York, NY, USA, 1966.

[41] M. A. Parra, S. Abrahams, K. Fabi, R. Logie, S. Luzzi, and S. D. Sala, "Short-term memory binding deficits in Alzheimers disease," Brain, vol. 132, no. 4, pp. 1057-1066, 2009.

[42] B. L. Chalfonte and M. K. Johnson, "Feature memory and binding in young and older adults," Memory and Cognition, vol. 24 , no. 4 , pp. 403-416, 1996.

[43] R. P. C. Kessels, D. Hobbel, and A. Postma, "Aging, context memory and binding: a comparison of "what, where and when" in young and older adults," International Journal of Neuroscience, vol. 117, no. 6, pp. 795-810, 2007.

[44] J. R. Brockmole, M. A. Parra, S. D. Sala, and R. H. Logie, "Do binding deficits account for age-related decline in visual working memory?" Psychonomic Bulletin and Review, vol. 15, no. 3 , pp. 543-547, 2008.

[45] S. T. Charles, M. Mather, and L. L. Carstensen, "Aging and emotional memory: the forgettable nature of negative images for older adults," Journal of Experimental Psychology: General, vol. 132, no. 2, pp. 310-324, 2003.

[46] L. A. Leigland, L. E. Schulz, and J. S. Janowsky, "Age related changes in emotional memory," Neurobiology of Aging, vol. 25, no. 8, pp. 1117-1124, 2004.

[47] M. Mather and M. Knight, "Goal-directed memory: the role of cognitive control in older adults' emotional memory," Psychology and Aging, vol. 20, no. 4, pp. 554-570, 2005.

[48] D. P. Smith, C. H. Hillman, and A. R. Duley, "Influences of age on emotional reactivity during picture processing," Journals of Gerontology-Series B Psychological Sciences and Social Sciences, vol. 60, no. 1, pp. P49-P56, 2005. 


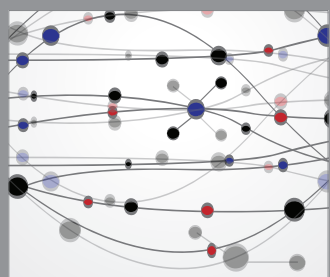

The Scientific World Journal
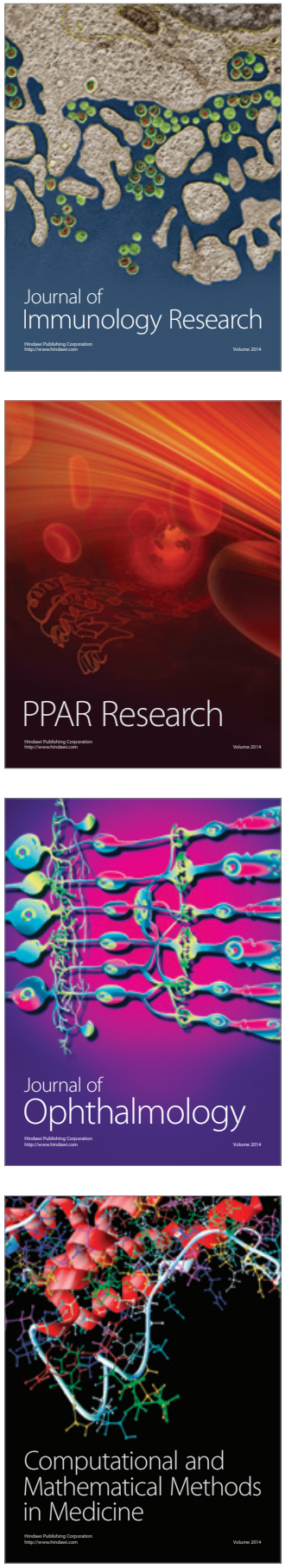

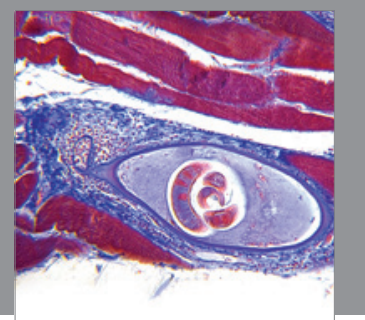

Gastroenterology

Research and Practice
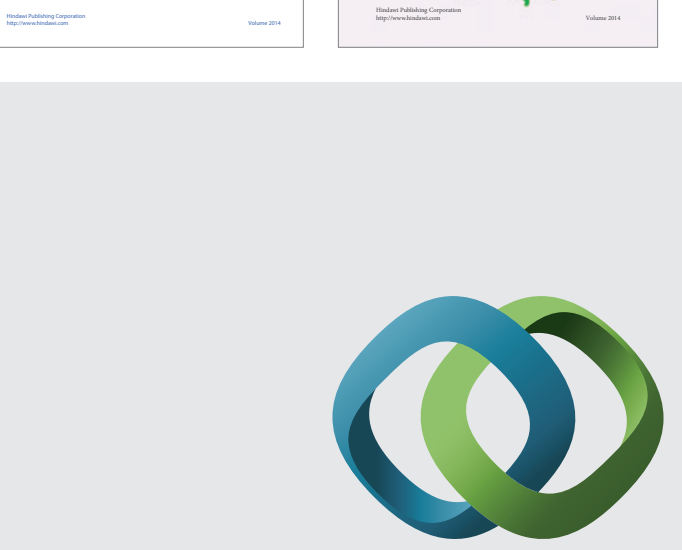

\section{Hindawi}

Submit your manuscripts at

http://www.hindawi.com
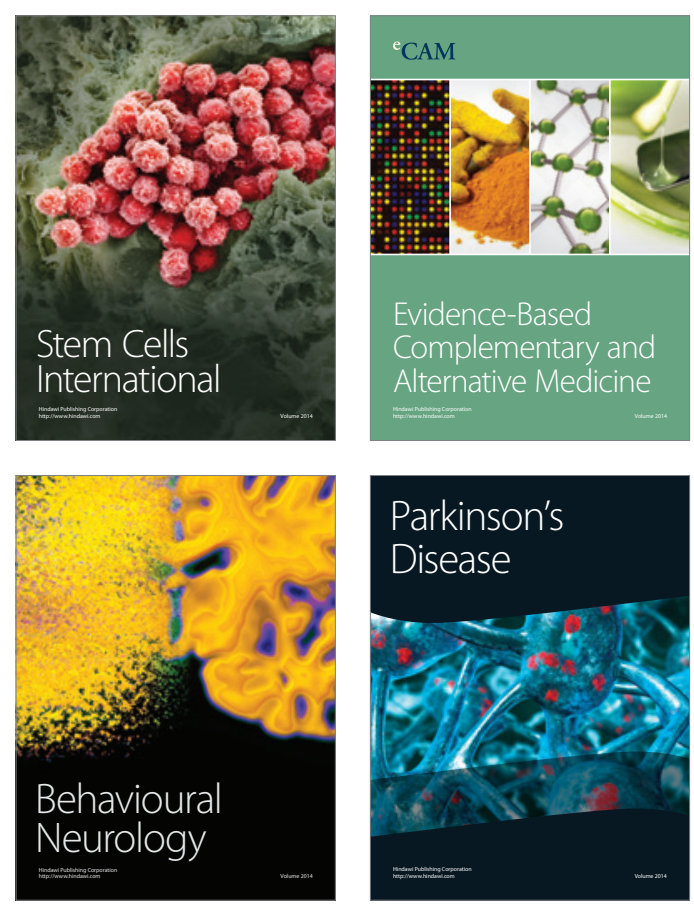

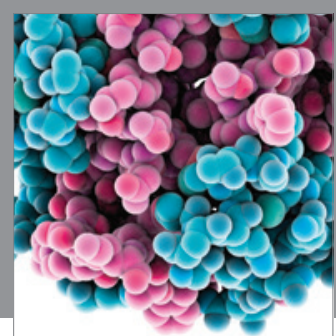

Journal of
Diabetes Research

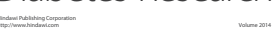

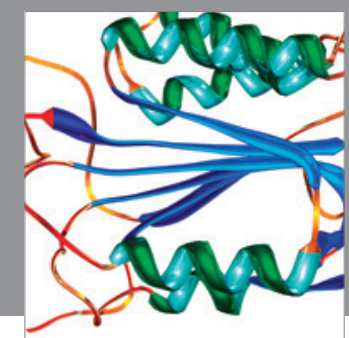

Disease Markers
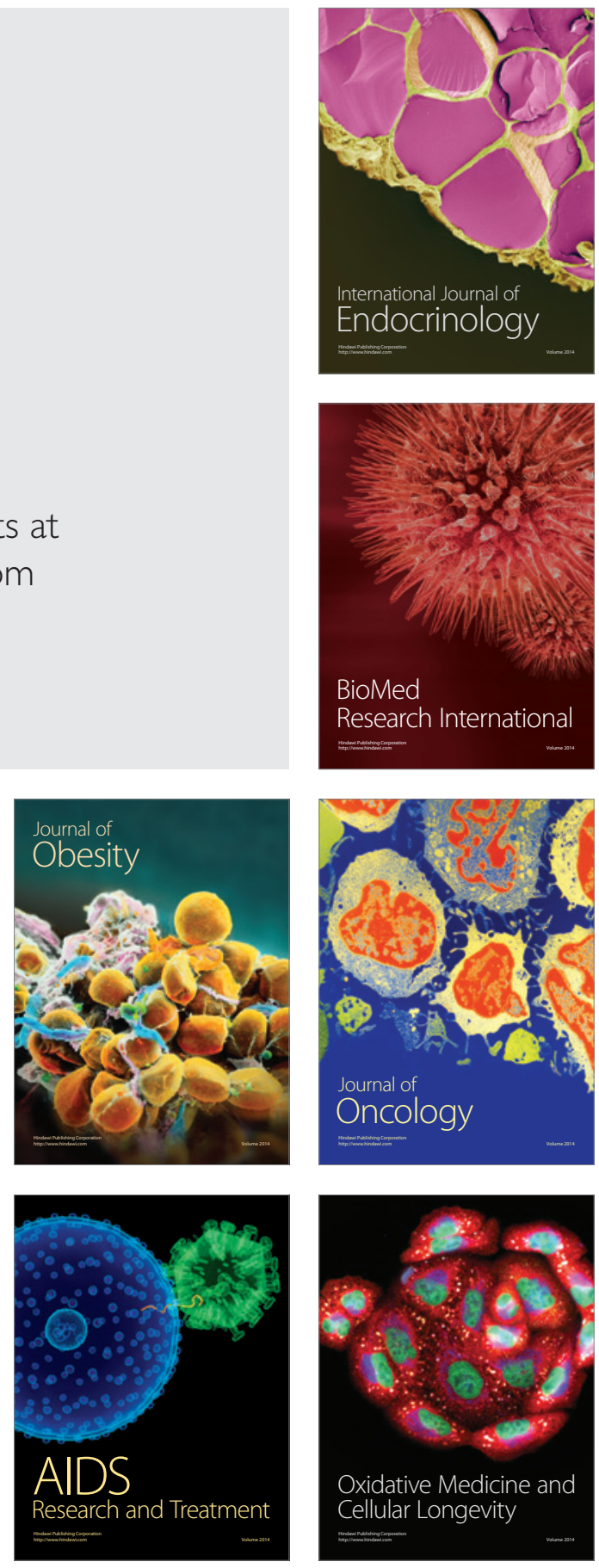